\title{
A clinico-pathological and cytological study of oral candidiasis
}

\author{
Kayo Kuyama ${ }^{1,2}$, Yan Sun ${ }^{1}$, Chieko Taguchi ${ }^{3}$, Hiroyasu Endo ${ }^{4}$, Masanobu Wakami ${ }^{5}$, \\ Masahiko Fukumoto ${ }^{6}$, Takanori Ito ${ }^{4}$, Hirotsugu Yamamoto ${ }^{1,2}$

\footnotetext{
${ }^{1}$ Department of Oral Pathology, Nihon University School of Dentistry at Matsudo, Chiba, Japan;

${ }^{2}$ Department of Diagnostic Pathology, Hospital of Nihon University School of Dentistry at Matsudo, Chiba, Japan;

${ }^{3}$ Department of Social Dentistry, Nihon University School of Dentistry at Matsudo, Chiba, Japan;

${ }^{4}$ Department of Oral Diagnosis, Nihon University School of Dentistry at Matsudo, Chiba, Japan;

${ }^{5}$ Department of Crown and Bridge Prosthodontics, Nihon University School of Dentistry at Matsudo, Chiba, Japan;

${ }^{6}$ Department of Laboratory Medicine for Dentistry, Nihon University School of Dentistry at Matsudo, Chiba, Japan.

Email: kuyama.kayo@nihon-u.ac.jp
}

Received 14 October 2011; revised 22 November 2011; accepted 3 December 2011.

\begin{abstract}
Candidiasis of the oral mucosa arises chiefly as a result of infection with Candida albicans. Many clinicopathological analyses of macroscopic findings have been described, although the clinical findings of oral candidiasis vary considerably and the conditions are complex. The present study analyzes the distribution, clinical, cytological and histological diagnoses of oral candidiasis, associated complex diseases and the diagnostic value of cytology. The ratio of Candida infection was $28.9 \%$ among 1551 study participants. Females were infected significantly more often than men $(p<0.01)$ and the affected age range was $60-79$ years $(61.0 \%, p<0.01)$. The predominantly affected areas were the tongue $(48.3 \%, \mathrm{p}<0.01)$ and gingiva $(20.0 \%, p<0.01)$, and occurrence at multiple loci was seen in $43(9.6 \%)$ patients. The typical clinical findings of oral candidiasis were ulcerative/erythematous lesions $(33.2 \%, p<0.01)$ and pseudomembranous candidiasis $(31.6 \%, p<0.01)$. A histopathological diagnosis of candidiasis based on biopsy specimens from 26 lesions in patients with Candida infection indicated by cytology was confirmed from cultures. The breakdown of a cytological to a definite diagnosis was 6 positive (SCC 4, verrucous carcinoma 1 , moderate to severe dysplasia 1), 6 suspected positive (mild dysplasia, 2; moderate to severe dysplasia, 2; papilloma, 1 and SCC, 1) and 14 negative (epulis, 3; papilloma, 3; granulation tissue, 2; fibrosis, 2 and others, 4). Exfoliative cytology can easily judge the presence of Candida species, although experience is necessary for the presumptive diagnosis of an oral mucosal disease. The application of exfoliative cytology using the Periodic acid-Schiff reaction is helpful for the earlier detection of oral candidiasis with various macroscopic findings.
\end{abstract}

Keywords: Candidiasis; Oral Exfoliative Cytology; Clinico-Pathology

\section{INTRODUCTION}

Candidiasis in the oral mucosa is usually caused by Candida albicans, which is an indigenous fungus in the oral cavity of healthy individuals. However, oral candidiasis can develop as a result of decreased host immunity, that is, as an opportunistic infection. The causes of oral candidiasis include being elderly or being an infant, having AIDS or diabetes, various drugs and local factors such as wearing dentures, steroid preparations and xerostomia. Many macroscopic findings of clinico-pathological analyses have been reported, although oral candidiasis has a variable clinical presentation and thus can be difficult to precisely diagnose. Exfoliative cytology to screen for oral mucosal disease has been performed for 30 years at our hospital and all specimens are checked for Candida. Additionally, the accuracy of detecting Candida by exfoliative cytology has already been proven by simultaneous culture testing [1].

The present study analyzes the distribution of oral candidiasis screened by exfoliative cytology according to sex, location, age and clinical findings. Clinical, cytological, histological diagnoses and complicated diseases associated with oral candidiasis and the effectiveness of cytology as a diagnostic tool are examined and discussed.

\section{MATERIALS AND METHODS}

We initially enrolled 1551 patients who presented mainly due to oral mucosal abnormalities, and who were diagnosed with a Candida infection by exfoliative cytology between April 2008 and March 2009 at the Department of Diagnostic Pathology at the Hospital of Nihon University School of Dentistry at Matsudo. All cytologi- 
cal specimens were examined by Papanicolaou (Pap) staining and the Periodic acid-Schiff (PAS) reaction, and reconfirmed by an internationally qualified cytological screener and three specialists in oral cytopathology. Candida infection was diagnosed when spores, pseudohyphae and/or mycelia were confirmed by PAS reaction. Candida infection in those with only detectable spores was confirmed by colony formation and culture (Nissui Pharmaceutical Co. Ltd., Tokyo, Japan). Six cases performed biopsy immediately among 12 cases in which the malignant tumor was suspected, cytological and clinically. The 6 remaining cases transferred to another hospital at the patient's requests. The 437 cases except these 12 cases were performed intraoral re-examination after they had removed Candida. Biopsies were obtained from those with consistent macroscopic findings and diseases were histopathologically diagnosed by three oral pathologists using hematoxylin and eosin staining (H.E.) to determine more complex diseases. Histopathological diagnoses followed the diagnostic criteria of the World Health Organization WHO [2]. Data were analyzed according to the age, location, clinical diagnosis, cytological diagnosis, histopathological diagnosis and disease complexity. The Ethics committee of Hospital of Nihon University School of Dentistry at Matsudo approved this study, and all patients provided written, informed consent to participate in all procedures associated with the study. All data were statistically analyzed using the Chisquare test (SPSS).

\section{RESULTS}

\subsection{Candida Infection Rate}

Among 1551 (male 576, female 975) patients who presented at our hospital with an oral mucosa disorder as the chief concern, 449 (28.9\%; male, 156 (34.7\%); female $293(65.3 \%))$ of them were infected with Candida.

\subsection{Distribution of Patients}

Significantly more women than men $(\mathrm{p}<0.01)$ were infected. The retrieval of information according to age excluded 2 men and 10 women of unknown age. Table 1 shows the age distribution of individuals with oral candidiasis. Most of the infected individuals were aged 60 79 years $(61.0 \%, \mathrm{p}<0.01)$. Table 2 lists the locations of oral candidiasis. Most lesions were on the tongue $(48.3 \%$, $\mathrm{p}<0.01)$, followed by the gingiva $(20.0 \%$, $\mathrm{p}<0.01)$, cheek $(11.1 \%)$, lips $(5.3 \%)$, palate $(4.2 \%)$ and oral floor $(1.3 \%)$. The tongue was further sub-classified as the dorsum $(43.8 \%, \mathrm{p}<0.01)$, margin $(40.5 \%, \mathrm{p}<0.01)$, apex $(7.4 \%)$, inferior aspect $(4.6 \%)$, root $(1.4 \%)$ and the entire tongue $5(2.3 \%)$. Maxillo-mandibular infections were located in the gingiva $(7.8 \% ; 4 / 90)$, lip $(8.3 \% ; 2 / 24)$,

${ }^{*}$ Cytological study of oral candidiasis right and left margin of the tongue $(5.7 \% ; 5 / 88)$, and cheek $(22.0 \% ; 11 / 50)$, and $43(9.6 \%)$ patients had lesions at multiple loci.

\subsection{Clinical Manifestations}

The clinical manifestations of patients with oral candidiasis (Table 3) comprised ulcerative/erythematous lesions $(33.2 \%, \mathrm{p}<0.01)$, pseudomembranous candidiasis $(31.6 \%, \mathrm{p}<0.01)$, white patch/leukoplakia $(12.2 \%)$, denture stomatitis $(8.9 \%)$, erythema $(7.1 \%)$, lichen planus $(4.2 \%)$, nodules $(2.2 \%)$ and angular cheilitis $(0.4 \%)$.

Table 1. Distribution of the patients of oral candidiasis.

\begin{tabular}{ccccccc}
\hline Age & No. & $\%$ & Male & $\%$ & Female & $\%$ \\
\hline $0-9$ & 2 & 0.4 & 0 & 0.0 & 2 & 0.7 \\
$10-19$ & 4 & 0.9 & 3 & 1.9 & 1 & 0.3 \\
$20-29$ & 6 & 1.3 & 2 & 1.3 & 4 & 1.4 \\
$30-39$ & 12 & 2.7 & 6 & 3.8 & 6 & 2.0 \\
$40-49$ & 28 & 6.2 & 14 & 9.0 & 14 & 4.8 \\
$50-59$ & 55 & 12.2 & 19 & 12.2 & 36 & 12.3 \\
$60-69$ & 102 & $22.7 * *$ & 30 & 19.2 & 72 & 24.6 \\
$70-79$ & 172 & $38.3 * *$ & 62 & 39.7 & 110 & 37.5 \\
$80-89$ & 46 & 10.2 & 17 & 10.9 & 29 & 9.9 \\
$90-99$ & 10 & 2.2 & 1 & 0.6 & 9 & 3.1 \\
Unknown & 12 & 2.7 & 2 & 1.3 & 10 & 3.4 \\
Total No. & 449 & 100.0 & 156 & 100.0 & 293 & 100.0 \\
\hline
\end{tabular}

**: A significant difference $(\mathrm{p}<0.01)$ was observed among all the age groups by chi-square test.

Table 2. The location of oral candidiasis.

\begin{tabular}{|c|c|c|c|c|c|}
\hline Location & No. & $\%$ & & No. & $\%$ \\
\hline \multirow[t]{3}{*}{ Gingiva } & 90 & 20.0 & Lower & 50 & $55.6 * *$ \\
\hline & & & Upper & 36 & $40.0 * *$ \\
\hline & & & Upper \& lower & 4 & $4.4 * *$ \\
\hline \multirow[t]{2}{*}{ Palate } & 19 & 4.2 & Hard & 16 & $84.2 * *$ \\
\hline & & & Soft & 3 & $15.8^{*}$ \\
\hline \multirow[t]{4}{*}{ Lip } & 24 & 5.3 & Upper & 12 & 50.0 \\
\hline & & & Lower & 8 & 33.3 \\
\hline & & & Angle & 2 & 8.3 \\
\hline & & & Upper \& lower & 2 & 8.3 \\
\hline Oral floor & 6 & 1.3 & & & \\
\hline \multirow[t]{7}{*}{ Tongue } & 217 & $48.3^{*}$ & Dorsum & 95 & $43.8 * *$ \\
\hline & & & Margin & 83 & $38.2 * *$ \\
\hline & & & Apex & 16 & 7.4 \\
\hline & & & Inferior aspect & 10 & 4.6 \\
\hline & & & Root & 3 & 1.4 \\
\hline & & & Right \& left margin & 5 & 2.3 \\
\hline & & & Entire tongue & 5 & 2.3 \\
\hline \multirow[t]{2}{*}{ Cheek } & 50 & $11.1 *$ & Cheek & 39 & $78.0 *$ \\
\hline & & & Right \& left cheek & 11 & $22.0 *$ \\
\hline Multiple & 43 & 9.6 & & & \\
\hline
\end{tabular}


Table 3. Clinical diagnoses of oral candidiasis.

\begin{tabular}{lccc}
\hline & & No. & $\%$ \\
\hline \multirow{3}{*}{ Acute forms } & Pseudo membranous & 142 & $31.6^{* *}$ \\
& Erythematous & 32 & 7.1 \\
\hline \multirow{4}{*}{ Chronic forms } & Leukoplakia & 55 & 12.2 \\
& Lichen planus & 19 & 4.2 \\
& Ulcerative/erythematous & 149 & $33.2^{* *}$ \\
& Nodular & 10 & 2.2 \\
\hline \multirow{3}{*}{$\begin{array}{l}\text { Candida-associated } \\
\text { lesions }\end{array}$} & Denture stomatitis & 40 & 8.9 \\
& Angular cheilitis & 2 & 0.4 \\
& Median rhomboid glossitis & 0 & 0.0 \\
\hline & Linear gingival erythema & 0 & 0.0 \\
\hline
\end{tabular}

**: A significant difference $(\mathrm{p}<0.01)$ was observed among all the age groups by chi-square test.

\subsection{Cytological and Histopathological Diagnoses}

Cytology with the PAS reaction detected Candida infection with $100 \%$ precision. That is, Candida infection was confirmed by mycelia growth in all of the cultures that tested positive by PAS. Table 4 shows the cytological and oral biopsy findings. Cytological diagnoses were negative in $395(88.0 \%)$, suspected positive in $39(8.7 \%)$ and positive in $15(3.3 \%)$ cases. The estimated negative diagnoses comprised inflammatory lesions $(67.9 \% ; 305 /$ $395)$ and hyperkeratosis $(20.0 \% ; 90 / 395)$, and the estimated positive diagnoses were verrucous $(0.2 \% ; 1 / 15)$ and squamous cell carcinoma (SCC, 3.1\%; 14/15). We histopathologically diagnosed 26 lesions from 24 patients in whom Candida infection was identified cytologically and confirmed by cultures. The breakdown of cytologically confirmed diagnoses is as follows. Six were confirmed as positive (SCC, $(n=4))$; verrucous carcinoma, $(n=1)$; moderate to severe dysplasia, $(n=1)$, 6 suspected positive with mild $(\mathrm{n}=2)$ and moderate to severe $(n=2)$ dysplasia; papilloma, $(n=1)$ and SCC (n $=1$ ), and 14 were confirmed as negative, and having epulis $(\mathrm{n}=3)$, papilloma $(\mathrm{n}=3)$, granulation tissue $(\mathrm{n}=$ $2)$, fibrosis $(n=2)$ and others $(n=4)$. Candida infection was accompanied by benign and malignant diseases in $20(76.9 \%)$ and $6(23.1 \%)$ patients, respectively.

\subsection{Treatment for Candida Infection}

The 437 patients in whom Candida infection was cytologically diagnosed were treated with an antifungal drug. These strategies resulted in the disappearance of the fungal mycelia from 432 cases $(98.9 \%)$.

\section{DISCUSSION}

Oral candidiasis is a common opportunistic infection in individuals with decreased immunity. Physiological factors

Table 4. Result of cytology, treatment and biopsy.

\begin{tabular}{|c|c|c|c|c|c|c|c|c|c|c|}
\hline Cytological diagnosis & No. & $\%$ & Estimate diagnosis & No & $\%$ & No. of A.D. & No. of C.C. ${ }^{* *}$ & No. of Biopsy & Definite diagnosis & No. \\
\hline \multirow[t]{8}{*}{ Negative } & 395 & 88.0 & Inflammatory change & 305 & 67.9 & 305 & 305 & 14 & Epulis & 3 \\
\hline & & & Hyperkeratosis & 90 & 20.0 & 90 & 86 & & Papilloma & 3 \\
\hline & & & & & & & & & Granulation tissue & 2 \\
\hline & & & & & & & & & Fibrosis & 2 \\
\hline & & & & & & & & & Fibro-epithelial polyp & 1 \\
\hline & & & & & & & & & Pyogenic granuloma & 1 \\
\hline & & & & & & & & & Capillry hemangioma & 1 \\
\hline & & & & & & & & & Sjögren syndrom & 1 \\
\hline \multirow[t]{4}{*}{ Suspicious of positive } & 39 & 8.7 & Dysplasia & 39 & 8.7 & 39 & 38 & 5 & Mild dysplasia & 2 \\
\hline & & & & & & & & & Moderate to severe dysplasia & 2 \\
\hline & & & & & & & & & Papilloma & 1 \\
\hline & & & & & & & & & Squamous cell carcinoma & 1 \\
\hline \multirow[t]{3}{*}{ Positive } & 15 & 3.3 & Verrucous carcinoa & 1 & 0.2 & 0 & 0 & 7 & Squamous cell carcinoma & 4 \\
\hline & & & $\mathrm{SCC}^{* * *}$ & 14 & 3.1 & 3 & 3 & & Verrucous carcinoma & 1 \\
\hline & & & & & & & & & Moderate to severe dysplasia & 1 \\
\hline
\end{tabular}

A.D.*: Antifungal drug; C.C.**: Cured Candidiasis; $\mathrm{SCC}^{* * *}$ : Squamous cell carcinoma. 
that predispose individuals to oral candidiasis comprise pregnancy, immune defects, drugs and malnutrition, and local factors including trauma, denture-associated problems and oral cancer [3]. Most reports have relied on macroscopic observation by dental clinicians, although many reports have described clinico-pathological studies of oral candidiasis. The present epidemiological study examined oral candidiasis detected by exfoliative cytology and by visible cultures. We also identified the value of oral exfoliative cytology for diagnosing oral candidiasis.

\subsection{Ratio of Candidiasis}

Candida species comprise the most common opportunistic fungal pathogens in humans, with $C$. albicans being the most prevalent cause of mucosal and systemic infection. C. albicans has been described as the most frequently encountered oral fungal commensal with detection rates of $40 \%$ to $65 \%$ in healthy adults [4]. The ratio of oral candidiasis in the present study was $28.9 \%$, which was similar to the reported $24 \%$ of outpatients at a dental clinic [5] and $24.5 \%$ in a review of eight publications [6]. On the other hand, the rate of candidiasis was $14.09 \%$ in a large-scale Brazilian study of 1586 randomly selected individuals [7]. However, the detection rates were very low when Candida infection was determined only from interviews and macroscopic observations.

\subsection{Epidemiological Features}

Infection rates were influenced by age and removable prostheses in a Brazilian study [7]. Age-matched statistical analysis in the present study found a significantly higher infection rate among 60 - 79-year-olds than in any other age group. Many factors have been investigated, such as an impaired host defense causing deceased Salivary flow [8], an increase in the morbidity rate of diabetes [9], wearing dentures [10], and taking medicine to treat chronic [11] and auto-immune diseases such as Sjögren syndrome [8]. The prevalence rate of oral candidiasis among children with oral mucosal diseases was the highest among those aged 0 - 12 years (28.4\%) [12], which was similar to findings from other countries [1315]. The ratio of 0 - 19-year-olds was very small in the present study. Rare symptoms of oral candidiasis in children might have been one of the causes. The higher prevalence of candidiasis among women in the present study is in agreement with the findings of other studies [16, 17]. In fact, $62.9 \%$ of the patients who presented with the chief concern of oral mucosal abnormalities were female in the present study Furthermore, xerostomia and autoimmune diseases that cause oral candidiasis are prevalent among women. Half of our patients with a Candida infection had lesions that were concentrated mostly on the dorsum and edges of the tongue. The dorsal sur- face is the main ecological niche for Candida in the oral cavity $[4,8,11,18]$. Presumably, chronic contact with dentures [10] and relationships with oral mucosal diseases such as leukoplakia and injuries might explain the high frequency of infections being located on their edges. Moreover, since multiple symptoms were quite abundant $(15.6 \%)$, we considered that factors such as age, drugs and immune defects were involved.

\subsection{Clinical Manifestations}

The clinical classification of candidiasis is highly complex because the findings are diverse. Therefore, we categorized candidiasis into acute, chronic and Candida-associated lesions based on Lakshman's classification [3]. We concentrated on the ulcerative/erythematous and pseudomembranous types. An inflammatory reaction was obvious in ulcerative/erythematous and Candidaassociated lesions. The hyphae of $C$. albicans tightly adhere to epithelial cells, and proteinases secreted by the hyphae damage the oral mucosa [19]. Schaller et al. assert that C. albicans proteinase causes tissue damage and increasing vascular permeability leads to an inflammatory reaction and clinical symptoms [20]. Chronic erythematous candidiasis is associated with corticosteroids, antibiotics and HIV infection. Dentists often treat stomatitis with triamcinolone acetonide, which is routinely available at Japanese drugstores and prolonged use of this drug can become problematic [21]. In addition, candidiasis can be a side effect of the inhaled steroids that are used to treat asthma [22] and allergosis. Because erythematous candidiasis is similar to a non-specific inflammatory reaction, it should be cytologically diagnosed as soon as possible. The reported ratio of denturerelated stomatitis (DRS) ranges from $11 \%$ to $67 \%$ [23]. A removable prosthesis is the most common cause of the growth and pathogenicity of Candida species [4,18,24]. Angular cheilitis is also associated with yeasts and bacteria in relation to wearing dentures [3]. Candida species proliferate by contact with the resin [10], and Candida counts significantly correlate with the intensity of denture plaque scores [25]. The rate of hyperplastic candidiasis that macroscopically presented like leukoplakia was $12.2 \%$ in the present study, and the malignant transformation rate was high [26]. Simultaneous Candida infection and several etiological factors seemed to play a role in malignant transformation [27]. Candida species were identified in $43.7 \%$ of the patients with lichen planus and leukoplakia in their oral cavities [28]. The reported prevalence of oral candidiasis varies between $8 \%$ and $94 \%$ in patients with advanced cancer, because of differences in diagnostic criteria, diagnostic methods and the study population [29]. Whether a condition depends only on a Candida infection or such infection with coexistent mucous membrane diseases should be determined as soon as possible. Macroscopic classification is 
limited as most epidemiological surveillance concerning oral candidiasis considers the candidiasis, DRS and cheilitis as separate entities $[17,30]$.

\subsection{Accuracy of Exfoliative Cytology}

Information about investigating Candida infection of the cervical area using Pap smears is very scarce [31]. Candida species have been detected based on the growth of hyphae on PAS smears [4,6]. The high accuracy of detecting oral candidiasis by exfoliative cytology was demonstrated here, as well as by others [1]. The cytological detection of candidiasis is simple, inexpensive, accurate and painless. As for oral candidiasis, it is clinically variegated to present the findings of leukoplakia or intractable ulcerative lesion, etc. In this result, the abbreviation half of oral candidiasis was occupied by these confusing clinical view. Therefore, discovery of candidiasis by cytology was useful for avoiding unnecessary biopsy. In addition, as a result of giving antifungal drug immediately, as for candidiasis detected by cytological diagnosis, the fungal mycelia disappeared with $98.9 \%$ of these cases. However, Candida can be over- and underdiagnosed when other mucosal diseases coexist, especially dysplasia and SCC. The size and shape of oral epithetlial cells infected with Candida significantly change $[1,32]$. Judgment of changes between atypia by Candida and dysplastic change should be required experience. In that case, judgment becomes possible by carrying out observation of a macro-scopic view, and exfoliative cytology after antifungal drug. In Japan, there is a custom which applies triamcinolone acetonide easily to stomatatis. Moreover, since triamcinolone acetonide is marketing, using it for a long period of time may be continued by a patient's judgment, and it tends to merge the side effects of the Candidal infection [33]. Cell atypia was observed in epithelial cells of the decubitus ulcer accompanied by the Candidal infection which uses triamcinolone acetonide for a long period of time, in this study. The observation of cytological specimen of intractable ulcer after the long-term application of triamcinolone acetonide should be carefully. The presence or absence of Candida species can be easily determined by exfoliative cytology, although experience is necessary for a presumptive diagnosis of oral mucosal disease. Exfoliative cytology using the PAS reaction enables earlier detection of oral candidiasis that presents with macroscopically variable symptoms.

\section{REFERENCES}

[1] Kuyama, K., Fifita, S.F., Mastumoto, T., Endo, H., Takahashi, H., Kato, T., Uehara, T. and Yamamoto, H. (2005) Clinico-cytological study of oral candidiasis. The Journal of the Japanese Society of Clinical Cytology, 44, 132-138.
[2] Barnes, L., Eveson, J.W., Reichart, P. and Sidransky, D. (2005) Pathology and genetics of head and neck tumours. IARC Press, Lyon.

[3] Samaranayake, L.P., Keung, L.W. and Jin, L. (2009) Oral mucosal fungal infections. Periodontology 2000, 49, 39-59. doi:10.1111/j.1600-0757.2008.00291.x

[4] Zegarelli, D.J. (1993) Fungal infections of the oral cavity. Otolaryngologic Clinics of North America, 26, 10691089.

[5] Samaranayake, L. (2009) Commensal oral candida in Asian cohorts. International Journal of Oral Science, 1, 2-5. doi:10.4248/IJOS.08006

[6] Scully, C., El-Kabir, M. and Samaranayake, L.P. (1994) Candida and oral candidosis: A review. Critical Reviews in Oral Biology \& Medicine, 5, 125-157.

[7] Carrard, V., Haas, A., Rados, P., Filho, M., Oppermann, R., Albandar, J. and Susin, C. (2011) Prevalence and risk indicators of oral mucosal lesions in an urban population from South Brazil. Oral Diseases, 17, 171-179. doi:10.1111/j.1601-0825.2010.01712.x

[8] Ergun, S., Cekici, A., Topcuoglu, N., Migliari, D.A., Külekçi, G., Tanyeri, H. and Isik, G. (2010) Oral status and Candida colonization in patients with Sjögren's syndrome. Medicine Oral, Pathogia Oral y Cirgía Bucal, 15, e310-e315.

[9] Motta-Silva, A.C., Aleva, N.A., Chavasco, J.K., Armond, M.C., França, J.P. and Pereira L.J. (2010) Erythematous oral candidiasis in patients with controlled type II diabetes mellitus and complete dentures. Mycopathologia, 169, 215-223. doi:10.1007/s11046-009-9240-6

[10] Da Silva, P.M., Acosta, E.J., De Rezende, P.L., Graeff, M., Spolidorio, D.M., Almeida, R.S. and Porto, V.C. (2011) Microscopical analysis of Candida albicans biofilms on heat-polymerised acrylic resin after chlorhexidine gluconate and sodium hypochlorite treatments. Mycoses, 23, epub ahead of print.

[11] Awano, S., Ansai, T., Takata, Y., Soh, I., Akifusa, S., Hamasaki, T., Yoshida, A., Sonoki, K., Fujisawa, K. and Takehara, T. (2008) Oral health and mortality risk from pneumonia in the elderly. Journal of Dental Research, 87, 334339. doi:10.1177/154405910808700418

[12] Majorana, A., Bardellini, E., Flocchini, P., Amadori, F., Conti, G. and Campus, G. (2010) Oral mucosal lesions in children from 0 to 12 years old: Ten years' experience. Oral Surgery, Oral Medicine, Oral Pathology, Oral Radiology \& Endodontics, 110, e13-e18. doi:10.1016/j.tripleo.2010.02.025

[13] Arnrup, K., Lundin, S.A. and Dahllöf, G. (1993) Analysis of paediatric dental services provided at a regional hospital in Sweden. Dental treatment need in medically compromised children referred for dental consultation. Swedish Dental Journal, 17, 255-259.

[14] Bánóczy, J., Rigó, O. and Albrecht, M. (1993) Prevalence study of tongue lesions in a Hungarian population. Community Dentistry and Oral Epidemiology, 21, 224-226. doi:10.1111/j.1600-0528.1993.tb00761.x

[15] Sedgley, C.M., Samaranayake, L.P., Chan, J.C. and Wei, S.H., (1997) A 4-year longitudinal study of the oral prevalence of enteric gram-negative rods and yeasts in Chinese children. Oral Microbiology and Immunology, 12, 183-188. doi:10.1111/j.1399-302X.1997.tb00377.x 
[16] Masipa, J.N., Hauman, C.H. and Raubenheimer, E.J. (1992) Oral carriage of Candida species in patients visiting the Medunsa Dental Clinic. The Journal of the Dental Association of South Africa, 47, 407-409.

[17] Jainkittivong, A., Aneksuk, V. and Langlais, R.P. (2002) Oral mucosal conditions in elderly dental patients. Oral Diseases, 8, 218-223. doi:10.1034/j.1601-0825.2002.01789.x

[18] De Oliveira, C.E., Gasparoto, T.H., Dionísio, T.J., Porto, V.C., Vieira, N.A., Santos, C.F. and Lara, V.S. (2010) Candida albicans and denture stomatitis: Evaluation of its presence in the lesion, prosthesis, and blood. International Journal of Prosthodontics, 23, 158-159.

[19] Akpan, A. and Morgan, R. (2002) Oral candidiasis. Postgraduate Medical Journal, 78, 455-459. doi:10.1136/pmj.78.922.455

[20] Schaller, M., Korting, H.C., Shäfer, W., Bastert, J., Chen W. and Hube, B. (1999) Secreted aspectic proteinase (Sap) activity contributes to tissue damage in a model of human oral candidosis. Molecular Microbiology, 34, 169180. doi:10.1046/j.1365-2958.1999.01590.x

[21] Kay, L.W. (1976) Corticosteroids in diseases of the oral mucosa. International Dental Journal, 26, 405-410.

[22] Fukushima, C., Shimoda, T., kawano, T., Tomari, W.S., Mitsuta, K., Obase, Y., matsuo, N., Matsuse, H. and Kohno, S. (2001) Effects of amphotericin B gargles on oral colonization of Candida albicans in asthmatic patients on steroid inhalation therapy. Respiration, 68, 465-470. doi:10.1159/000050552

[23] Senpuku, H., Sogame, A., Inosita, E., Tsuha, Y., Miyazaki, H. and Hanada, N. (2003) Systemic diseases in association with microbial species in oral biofilm from elderly requiring care. Gerontology, 49,301-309. doi:10.1159/000071711

[24] Abaci, O., Ualiki-Uztan, A., Ozturk, B., Toksavul, S., Ulusoy, M. and Boyacioglu, H. (2010) Determining Candida spp. incidence in denture wearers. Mycopathologia,
169, 365-372. doi:10.1007/s11046-010-9275-8

[25] Budtz-Jłrgensen, E., Mojon, P., Banon-Clément J.M. and Baehni, P. (1996) Oral candidosis in long-term hospital care: comparison of edentulous and dentate subjects. Oral Diseases, 2, 285-290.

[26] Walker, D.M. and Arendorf, T. (1990) Oral candidosis. In: Samaranayake, L.P. and MacFarlane, T.W., Eds., Candidal Leukoplakia, Chronic Multifocal Candidosis and Median Rhomboid Glossitis, Butterworth, London, 184-199.

[27] Bánóczy, J. (1977) Follow-up studies in oral leukoplakia. Journal of Maxillofacial Surgery, 5, 69-75. doi:10.1016/S0301-0503(77)80079-9

[28] Lipperheide, V., Quindós, G., Jiménez, Y., Pontón, J., Bagán-Sebastián, J.V. and Aguirre, J.M. (1996) Candida biotypes in patients with oral leukoplakia and lichen planus. Candida biotypes in leukoplakia and lichen planus. Mycopathologia, 134, 75-82. doi:10.1007/BF00436868

[29] Davies, A.N., Brailsford, S.R., Beighton, D., Shorthose, K. and Stevens, V.C. (2008) Oral candidosis in community-based patients with advanced cancer. Journal of Pain and Symptom Management, 35, 508-514. doi:10.1016/i.jpainsymman.2007.07.005

[30] Jainkittivong, A., Aneksuk, V. and Langlais, R.P. (2010) Oral mucosal lesions in denture wearers. Gerodontology, 27, 26-32. doi:10.1111/j.1741-2358.2009.00289.x

[31] Zakout, Y.M., Salih, M.M. and Ahmed, H.G. (2011) Frequency of Candida species in Papanicolaou smears taken from Sudanese oral hormonal contraceptives users. Biotechnic \& Histochemistry, 14, Epub Ahead of Print.

[32] Loss, R., Sandrin, R., frança, B.H., De Azevedo-Alanis, L.R., Grégio, A.M., Machado, M.Â. and Lima, A.A. (2011) Cytological analysis of the epithelial cells in patients with oral candidiasis. Mycoses, 54, e130-e135. doi:10.1111/j.1439-0507.2009.01857.x

[33] Vogt, F.C. (1979) The incidence of oral candidiasis with use of inhaled corticosteroids. Ann Allergy, 43, 205-210. 